\title{
Identification of Urinary Biomarkers Related to Cisplatin- Induced Acute Renal Toxicity Using NMR-Based Metabolomics
}

\author{
He Wen ${ }^{1}$, Hye-ji Yang ${ }^{1}$, Myung-Joo Choi ${ }^{2}$, Hyuk Nam Kwon ${ }^{1}$, Min Ah Kim², Soon-Sun Hong ${ }^{2}$ and Sunghyouk \\ Park $^{1, *}$ \\ ${ }^{1}$ Department of Biochemistry, Inha University Hospital and Center for Advanced Medical Education by BK21 Project, \\ College of Medicine, Inha University, Incheon 400-712, \\ ${ }^{2}$ Department of Biomedical Sciences, Inha University Hospital and Center for Advanced Medical Education by BK21 Project, \\ College of Medicine, Inha University, Incheon 400-712, Republic of Korea
}

\begin{abstract}
Cisplatin is widely used for various types of cancers. However, its side effects, most notably, renal toxicity often limit its clinical utility. Although previous metabolomic studies reported possible toxicity markers, they used small number of animals and statistical approaches that may not perform best in the presence of intra-group variation. Here, we identified urinary biomarkers associated with renal toxicity induced by cisplatin using NMR-based metabolomics combined with Orthogonal Projections to Latent Structures-Discriminant Analysis (OPLS-DA). Male Sprague-Dawley rats $(\mathrm{n}=22)$ were treated with cisplatin $(10 \mathrm{mg} / \mathrm{kg}$ single dose), and the urines obtained before and after treatment were analyzed by NMR. Multivariable analysis of NMR data presented clear separation between non-treated and treated groups. The OPLS-DA statistical results revealed that 1,3-dimethylurate, taurine, glucose, glycine and branched-chain amino acid (isoleucine, leucine and valine) were significantly elevated in the treated group and that phenylacetylglycine and sarcosine levels were decreased in the treated group. To test the robustness of the approach, we built a prediction model for the toxicity and were able to predict all the unknown samples $(n=14)$ correctly. We believe the proposed NMR-based metabolomics with OPLS-DA approach and the resulting urine markers can be used to augment the currently available blood markers.
\end{abstract}

Key Words: Cisplatin, Toxicity, NMR, OPLS-DA

\section{INTRODUCTION}

Cisplatin is the founding member of the platin-group drugs that has platinum metal and ammonium group. Since its first approval in 1978 by US Food and Drug Administration, it has been widely used for various types of cancer such as lung, ovarian, lymphomas, breast and bladder cancers (Smith and Talbot, 1992; von der Maase et al., 2000; Crino et al., 2001; Muggia, 2009). The mechanism of anticancer effect is cytotoxicity due to DNA cross-linking, oxidative damages and apoptosis (Gong et al., 1999; Pruefer et al., 2008). Due to these cytotoxic effect, cisplatin kills not only cancer cells but also normal cells, resulting in undesirable effects in various tissues including kidney, nerves, ear and gastroenteric ones (Loehrer and Einhorn, 1984). Among these, the nephrotoxicity is most common and can be a cause for the cessation of the drug therapy (Arany and Safirstein, 2003; Yao et al., 2007). Typically, Blood
Urea Nitrogen (BUN), and blood creatinine are checked to monitor the expression of renal toxicity. In addition, hydration and diuretic measures are taken to minimize possible kidney damages. Still, BUN and creatinine are measured from blood and are late stage kidney functional markers (Hewitt et al., 2004; Davis and Kramer, 2006). Therefore, alternative urine markers may aid clinicians to decide the degree of the toxicity more conveniently.

Evaluation of drug-induced toxicity can be performed in various ways. Direct observation of tissues of interest would provide ultimate answer, but it is often inconvenient or impossible to obtain the tissue without sacrificing the animals. Therefore toxicological markers have been explored that reflect the status of organs. Metabolomics have emerged as a very promising tool for finding and assessing non-invasive markers for drug induced toxicity (Nicholson et al., 1999; Nicholson et al., 2002; Ebbels et al., 2007), because it uses bio-fluids, such as www.biomolther.org

Open Access DOI: 10.4062/biomolther.2011.19.1.038

pISSN: 1976-9148 elSSN: 2005-4483

Copyright $\odot 2011$ The Korean Society of Applied Pharmacology
Received Sep 15, 2010 Revised Sep 15, 2010 Accepted Oct 11, 2010

*Corresponding Author
E-mail: spark@inha.ac.kr
Tel: +82-32-890-0935, Fax: +82-32-884-6726 
blood and urine, to evaluate changes of levels in endogenous small molecules upon drug challenge. It has not only been applied to evaluating drug-induced toxicity, but also to predicting toxicity before drug treatment, opening doors to personalized drug treatment (Clayton et al., 2006; Clayton et al., 2009). With its other application to disease diagnosis and evaluation of prognosis after therapies (Wishart, 2005; Wen et al., 2010b) it is rapidly becoming a valuable tool in many fields of biomedical sciences.

In evaluating kidney toxicities by cisplatin, metabolomics studies were also reported recently (Portilla et al., 2006; Boudonck et al., 2009). One study used NMR combined with Principal Component Analysis (PCA), and another used Mass spectroscopy with Classification and Regression Trees (CART) or logistic regression. Still, these work used less than seven animals for the statistical analysis, and therefore, there is a need to confirm the results with a larger number of animals. Moreover, for the statistical approaches, PCA, although it has been used in many metabolomics studies, may not give the best definitive markers for a known property, treated group vs. non-treated group in this case, as it is a non-supervised multivariate analysis. CART or logistic regression may not perform best in the presence of large intra-group variation. In comparison, Orthogonal Projections to Latent StructuresDiscriminant Analysis (OPLS-DA), is a supervised discrimination method that can deal with large intra-group variation, and thus provide markers that can be interpreted straightforwardly (Bylesjo et al., 2006; Wiklund et al., 2008). In addition, the approach gives a prediction model that can be used to test unknown samples for the presence of the property of interest.

Here, we employed NMR-based metabolomics combined with OPLS-DA multivariate analysis to distinguish treated and non-treated group and find associated toxicity markers. Our multivariate model robustly predicted the unknown samples for the presence of toxicity. We believe the proposed NMRbased metabolomics with OPLS-DA approach and the resulting urine markers can be used to augment the currently available blood markers and be applied to assess toxicities associated with other drugs.

\section{MATERIALS AND METHODS}

\section{Materials and animals}

Cisplatin was obtained from Sigma Chemical Company (St. Louis, MO). Male Sprague-Dawley rats were obtained from Orient Bio (Sungnam, Korea) at age of 6-7 weeks old. The animals were kept in polypropylene cages with stainless steel grid. Food and water were available freely. The animal rooms were well controlled for balanced humidity and temperature condition with $12 \mathrm{hr}$ light/dark cycle. Animal care and all experimental procedures were conducted in accordance with the guide for animal experiments edited by the Korea Academy of Medical Science, Declaration of Helisinki principle, and approved by the institutional review board. All animal experiments were done at the Inha University Medical school Animal experiment center (Incheon, Korea). Individual animal data are listed in Table S1.

\section{Cisplatin administration and urine sample collection}

Rats were randomly divided into dose group and control group. The dose group $(\mathrm{n}=22)$ was given $10 \mathrm{mg} / \mathrm{kg}$ of cisplatin in saline and the control group $(n=8)$ was treated with just saline by an intraperitoneal injection. Urine samples were collected before (-24 to $0 \mathrm{hrs}$ ) and after treatment (72 to $96 \mathrm{hrs}$ ) into an ice-cooled jar with a metabolic cage. The collected urine samples were frozen and stored at $-80^{\circ} \mathrm{C}$ until metabolic analysis.

\section{Hematology markers and histological study}

After 96 hour treatment, the blood samples were collected using heart puncture before sacrifice. Blood samples were allowed to clot at room temperature and serum was obtained by centrifugation at $13,000 \mathrm{rpm}$ for $10 \mathrm{~min}$ for clinical chemistry (BUN and creatinine levels). The kidneys were collected immediately after blood collection, washed with saline buffer and then fixed in $10 \%$ formalin. Histological sections of the kidney were stained with hematoxylin and eosin (H\&E) stains. Statistical analysis was performed using an unpaired Student's $t$-test on SPSS software for Windows (Version 10.0; SPSS, Chicago, IL, USA).

\section{NMR measurement}

NMR spectra of the urine samples were measured with an NMR spectrometer (Bruker Biospin, Avance 500) operating at a proton NMR frequency of $500.13 \mathrm{MHz}$. The acquisition parameters were the same as previously reported (Kang et al., 2008a; Wen et al., 2010b). Two-dimensional NMR spectra were also acquired for identification and structural analysis of the metabolites with a $900 \mathrm{MHz}$ Bruker spectrometer. For proton correlations, Double Quantum Filtered Correlation Spectroscopy (DQF-COSY) data and total correlation spectroscopy (TOCSY) were obtained. For proton and carbon correlation, Heteronuclear Multiple Bond Correlation (HMBC) spectrum and Heteronuclear Single Quantum Coherence (HSQC) spectrum were measured. The experimental parameters are same as previously reported (Kang et al., 2008a; Kang et al., 2008b). We also used Chenomx (Spectral database; Edmonton, Alberta, Canada) for identification of the metabolites. This study used the NMR facility at Korea Basic Science Institute, which is supported by Bio-MR Research Program of the Korean Ministry of Science and Technology (E29070).

\section{Multivariate data analysis}

Raw NMR data were Fourier transformed, phase corrected, baseline corrected manually, and normalized against total integration values, $0.025 \%$ TSP, and converted to an ascii text file, then binned using in-house written Perl software as previously described (Wen et al., 2010a). For statistical analysis, water and urea regions were excluded. Multivariate statistical analysis was performed using the following softwares: Matlab (MathWorks, Natick, MA), SIMCA-P version 11.0 (Umetrics, Sweden). Orthogonal projections to latent structure-discriminant analysis (OPLS-DA) was performed with one predictive and four orthogonal component (Trygg and Wold, 2002; Bylesjo et al., 2006). 1D projection of Statistical Correlation Spectroscopy (STOCSY) was built by overlaying the colorcoded correlation values on to the OPLS-DA variable plot (Cloarec et al., 2005; Maher et al., 2009; Sands et al., 2009). 


\section{RESULTS}

\section{Biochemical parameters}

To evaluate the toxic effects of cisplatin, we measured the serum levels of BUN and creatinine which are widely used as hematological markers of nephrotoxicity. Both BUN (215.5 \pm $42.7 \mathrm{mg} / \mathrm{dl}$, mean \pm S.D., $\mathrm{n}=22)$ and creatinine $(4.8 \pm 0.9 \mathrm{mg} /$ dl, mean \pm S.D., $n=22$ ) were significantly elevated by 20 and 10 folds, respectively (Fig. 1A, B in the dose group compared to the control group (BUN: $10.7 \pm 2.1 \mathrm{mg} / \mathrm{dl}$, creatinine: 0.4 $\pm 0.03 \mathrm{mg} / \mathrm{dl}$, mean \pm S.D., $\mathrm{n}=8$; see Fig. $\mathrm{S} 1$ for bodyweight changes). Elevated BUN and creatinine indicated that treatment of rats with cisplatin increase the renal toxicity.

\section{Histopathological observations}

To confirm that the increased levels of the blood toxicity markers are actually correlated with damages in the kidney tissue, we performed the histopathological analysis of kidneys. As shown in Fig. 1C, H\&E staining of the kidney showed that the dose group exhibit significant kidney tissue damages represented by irregular tubular and cellular shapes which were not seen in the control group. Therefore, both histopathological observations and elevated BUN/creatinine levels indicate that significant kidney toxicity occurred when rats were given $10 \mathrm{mg} / \mathrm{kg}$ cisplatin.

\section{${ }^{1} \mathrm{H}-\mathrm{NMR}$ analysis of urine samples}

${ }^{1} \mathrm{H}-\mathrm{NMR}$ spectral analysis was performed on urine samples from both control and cisplatin treated groups. Before biomarker identification and characterization, principal component analysis (PCA) was performed to see the differences between individual samples. We found that the spectral characteristics of the pre-treatment time point (-24 to $0 \mathrm{hrs}$ ) of the dose group were not different from those of the post-treatment time point (72 to $96 \mathrm{hrs)}$ ) of the control group (data not shown), indicating that the variation of the metabolites between these groups are safely ignored. Therefore, we used the pre-treatment urine of the dose group as non-treated sample for the subsequent multivariate analysis. The representative ${ }^{1} \mathrm{H}$ NMR spectra of non-treated and treated urine samples are shown in Fig. 2. We identified 31 prominent metabolites in representative spectra using Chenomx and two-dimensional NMR spectra (Table S2), but their differential distribution between the non-treated and treated group could not be easily established due to the intra-group variation. In addition, although some differences can be characterized by simply visual inspection, the total correlation among the entire contents of the metabolites was not possible. Therefore, we applied a supervised multivariate statistical analysis to investigate variation of metabolites and to identify the biomarkers for the toxicity.

\section{OPLS-DA multivariate analysis}

We employed OPLS-DA multivariate statistical analysis, because it can distinguish groups in the presence of large intra-group variation (Bylesjo et al., 2006; Kang et al., 2008a). The differentiation model for classifying the animals between non-treated and treated group was effectively achieved using one predictive and four orthogonal components with very high reliability. As shown in Fig. 3, the non-treated and treated group can be separated clearly by the first predictive component, and the model had an overall goodness of fit, $R^{2}(Y)$, of $98.8 \%$ and an overall cross-validation coefficient, $\mathrm{Q}^{2}(\mathrm{Y})$, of $96.8 \%$. Out of the overall $R^{2}(X)$ value of $0.83,63 \%$ was structured, but uncorrelated to the response, and $20 \%$ was predic-
A
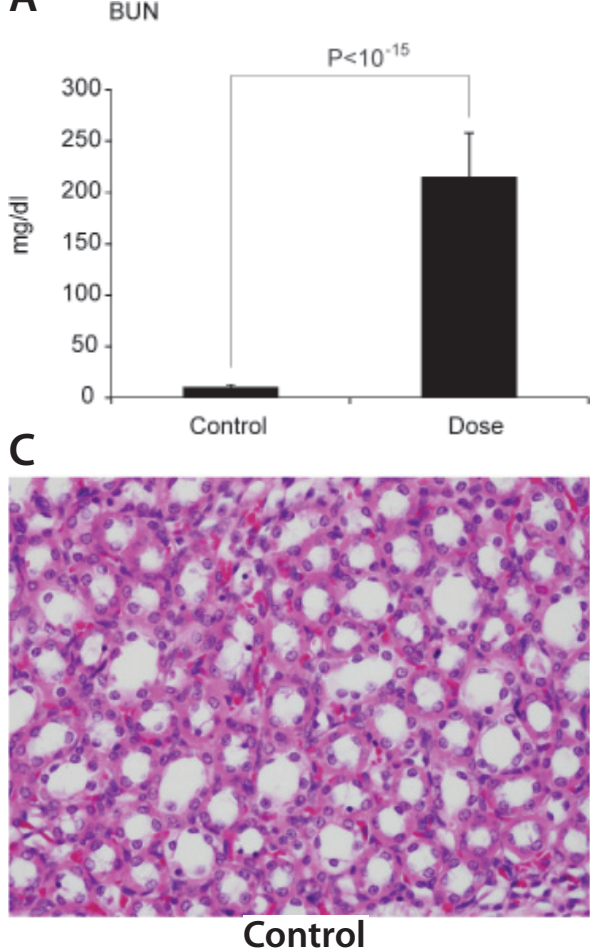

B

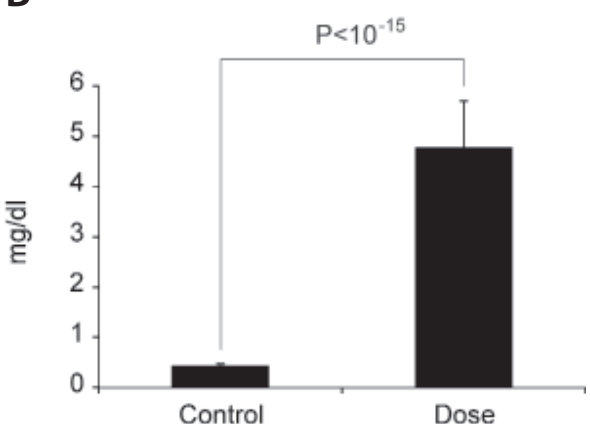

Fig. 1. Cisplatin-induced toxicity evaluated by hematology markers and histology. The control group was given just saline, and the dose group was given $10 \mathrm{mg} / \mathrm{kg}$ of cisplatin in saline. BUN and Creatinine levels were measured immediately after sacrificing the animals. (A, B) Changes in BUN and Creatinine levels, respectively. Data are expressed as mean \pm S.D. (C) Histological evaluation of the cisplatininduced kidney toxicity. The kidneys were harvested and fixed in 10\% formaldehyde. Histological sections of the kidney were stained with $\mathrm{H} \& \mathrm{E}$. 
tive, that is, responsible for the class separation. The complete separation between non-treated and treated group in OPLSDA model is consistent with the result of the above blood biochemical analysis (Table S1). To identify marker metabolites that contribute to this separation, we applied Statistical Total Correlation Spectroscopy (STOCSY) analysis on the OPLSDA modeled-data. Based on the STOCSY analysis (Fig. 4), we selected several metabolites according to the orders of contribution to the separation, which could be considered as toxicity biomarkers. They are 1,3-dimethylurate (3.4212 ppm), taurine (3.2415 ppm), glucose (3.487 ppm), glycine (3.5528 ppm) and branched-chain amino acid (isoleucine, leucine and valine) whose levels were significantly elevated in the treated group, and phenylacetylglycine (3.6667 ppm) and sarcosine (3.5834 ppm) whose levels were highly decreased in the treated group. To confirm the actual biased presence of these markers, we constructed plots of the relative amounts of these metabolites using independent Student's-t test (Fig. 5). The result confirms that all of these metabolites are significantly different in the two groups with $p$-values less than 0.0001 .
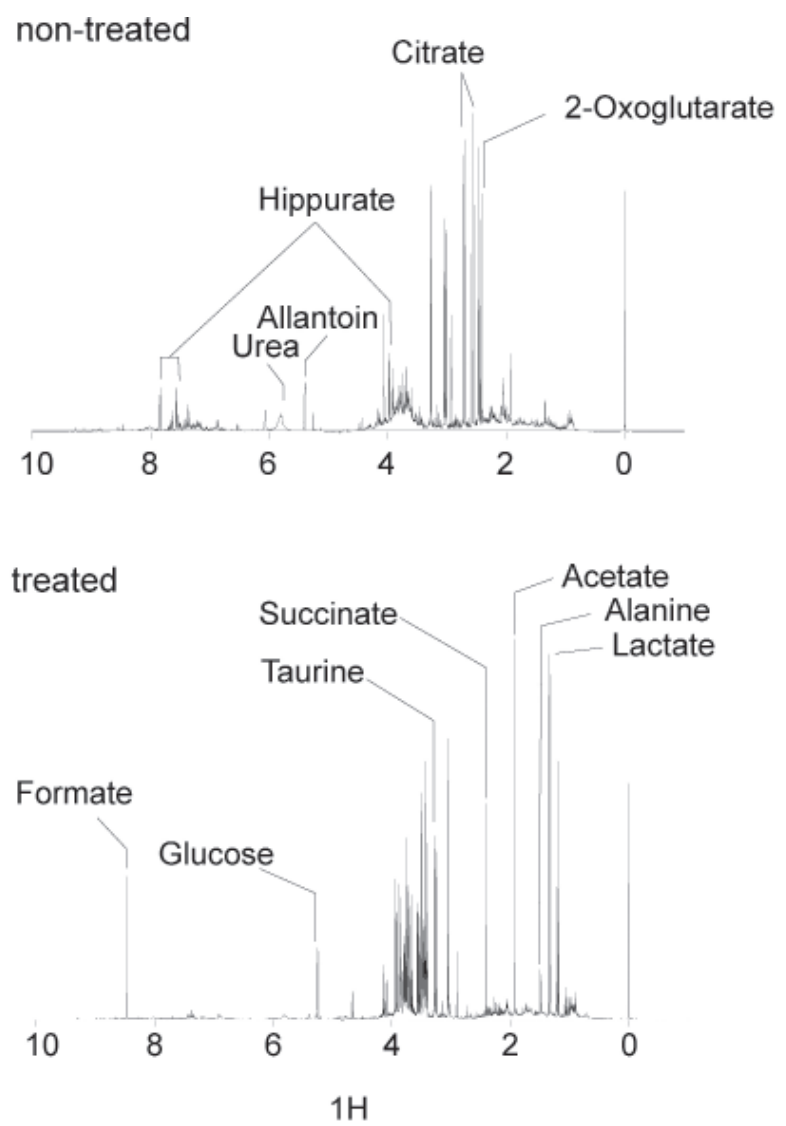

Fig. 2. Representative urine NMR spectra of non-treated and treated group. Spectra for the non-treated and treated group are in the top and in the bottom, respectively. Metabolite peaks were assigned using Chenomx (Spectral database; Edmonton, Alberta, Canada) and ultra high-field (900 MHz), two-dimensional NMR spectra (see text). The spectra were taken for samples in $500 \mu \mathrm{l}$ of $\mathrm{D}_{2} \mathrm{O}$ and urine mixture containing $200 \mathrm{mM}$ sodium phosphate $(\mathrm{pH}$ 7.4 ) and $0.025 \%$ TSP as a chemical shift reference.

\section{Toxicity prediction}

For the practical usability of the OPLS-DA model and the markers, a quantitative assessment on the predictability of the obtained model is very important, although it may not be performed in the PCA-based metabolomics literature. The prediction can also serve as the most rigorous validation of the differentiation model. To do that, we performed prediction analysis by randomly taking out as much as $30 \%$ of the samples from the entire dataset (test set), and building a new OPLS-DA model with only the remaining samples (training set). Then the test set was predicted for their class membership (non-treated vs. treated) the prediction model based only on the training set. As shown in Fig. 6, all 14 test samples (7 non-treated samples and 7 treated samples) were predicted correctly with a priori cut-off value of 0.5 , which confirms the robustness of the OPLS-DA model.

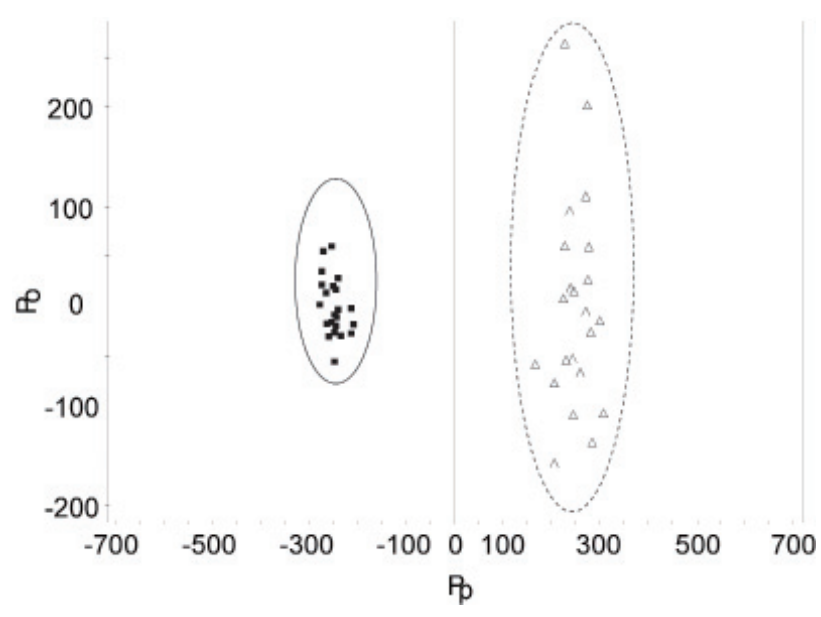

Fig. 3. Differentiation of non-treated and treated group using OPLS-DA multivariate analysis. Orthogonal projections to latent structure discriminant analysis (OPLS-DA) score plot of the nontreated and treated group. Filled box: non-treated group, Open triangle: treated group. The model was established using one predictive and four orthogonal components.

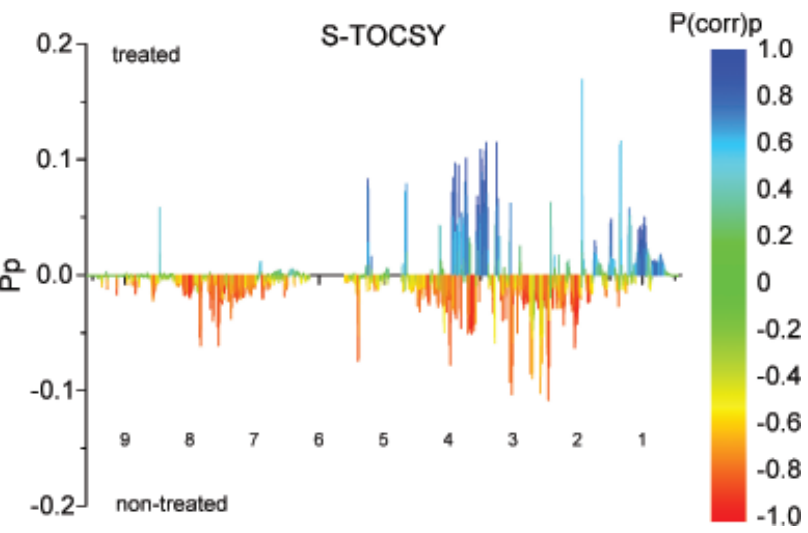

Fig. 4. Variable contributions from 1D projection of the statistical total correlation spectroscopy (STOCSY). The Pp represents modeled covariation and $\mathrm{P}$ (corr)p represents modeled correlation, which are shown in the color scale on the right. 
A
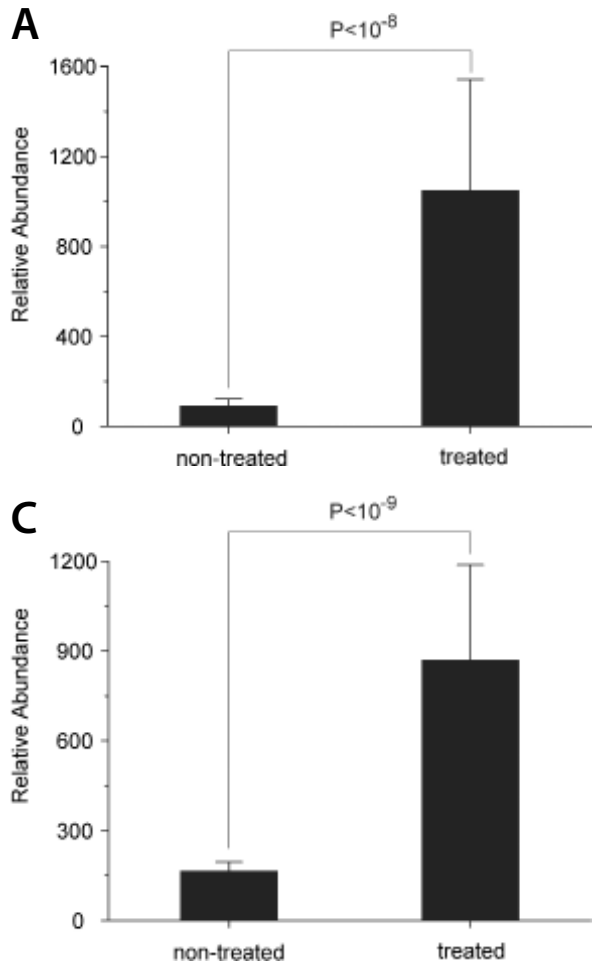

E

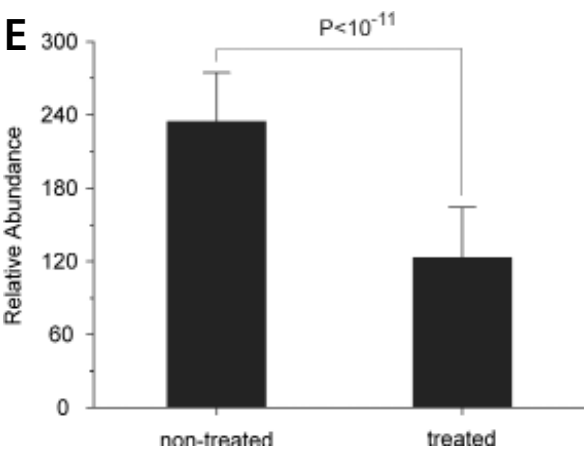

B

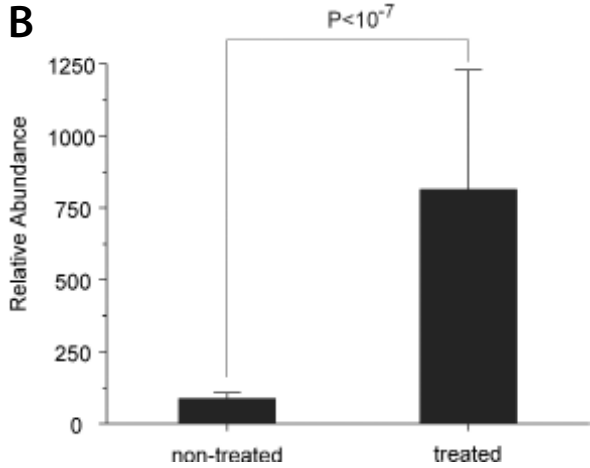

D
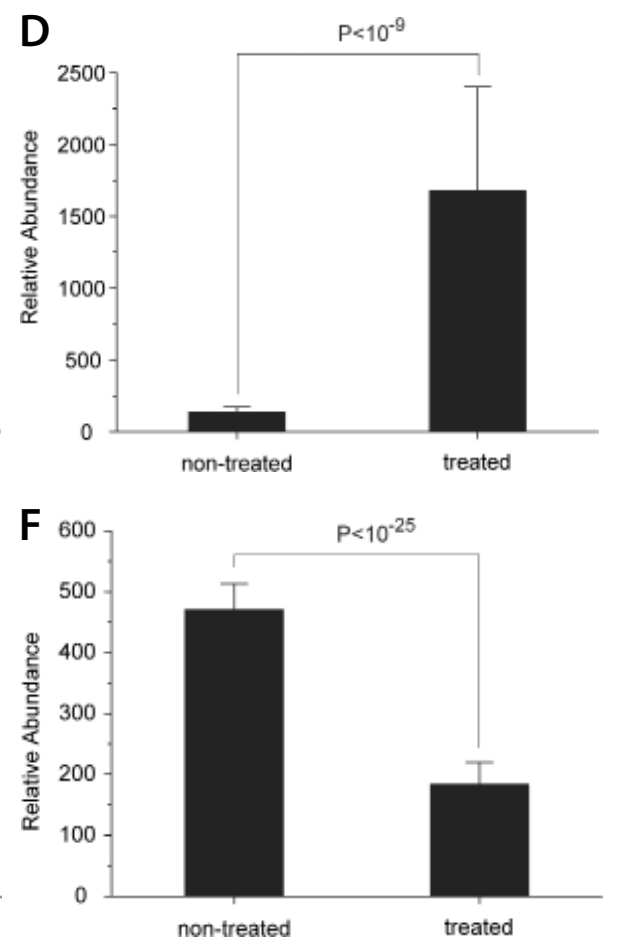

Fig. 5. Distribution of the marker metabolites in non-treated and treated group. Student's $t$-test of the relative distribution of the marker signals for the non-treated and treated group. The resulting $p$-values are indicated, and all the signals showed statistical significance with $p<0.0001$. (A) 1,3-dimethylurate: $3.4212 \mathrm{ppm}$, (B) Taurine: 3.2415 ppm, (C) Glycine: 3.5528 ppm, (D) Glucose: $3.487 \mathrm{ppm}$, (E) Sarcosine: $3.5834 \mathrm{ppm},(\mathrm{F})$ Phenylacetylglycine: $3.6667 \mathrm{ppm}$.

\section{DISCUSSION}

In this study, we assessed kidney toxicity induced by cisplatin using NMR-based metabolomics coupled with OPLSDA multivariate analysis, and presented the markers and the prediction model of the toxicity. In the course of the preliminary experiment, we observed that about a quarter of animals does not show appreciable signs of toxicity, indicative of idiopathic toxic responses of cisplatin in SD rats. The detailed analysis of the idiopathic toxicity will be published elsewhere, and we focused on those animals which developed toxicity in this current study.

Out of the markers elevated with the kidney damages, glucose and branched amino acids have been reported previously for cisplatin induced toxicity (Portilla et al., 2006; Boudonck et al., 2009). Here, we identified additional markers, glycine and taurine. Taurine is a sulfur-containing compound and its elimination through kidney is regulated by $\beta$-amino acid transport system present in the luminal brush boarder membrane (Rozen et al., 1979). Taurine is normally present in rat urine, but is also extensively re-absorbed through brush border mem- brane of the kidney cortex, and therefore, the kidney damage is expected to increase its urinary excretion (Maxuitenko et al., 1997). Glycine is the smallest amino acid and passes through glomerulus due to the small sizes. However, it is extensively re-absorbed by proximal tubule, and thus minimally present in normal urine. Therefore, elevated levels of glycine are also indicative of tubular damage. Taken together, the higher levels of taurine and glycine suggest that tubular re-absorption function is significantly damaged.

As statistical differentiation, we used OPLS-DA approach, whereas a previous NMR study used PCA and another MS study used t-test followed by either CART or logistic regression (Portilla et al., 2006; Boudonck et al., 2009). Although each approach has its own merits and shortcomings, our approach gave very good performance in classifying the unknown test set, predicting correctly all the test set data, up to $30 \%$ of the entire data set at 3 day time point. PCA is generally used for assessing sample-to-sample variation rather than classifying unknowns into established categories (Kang et al., 2008a). T-test of the entire variables has been used in mass spectrometry-based metabolomics (Sreekumar et al., 2009). 


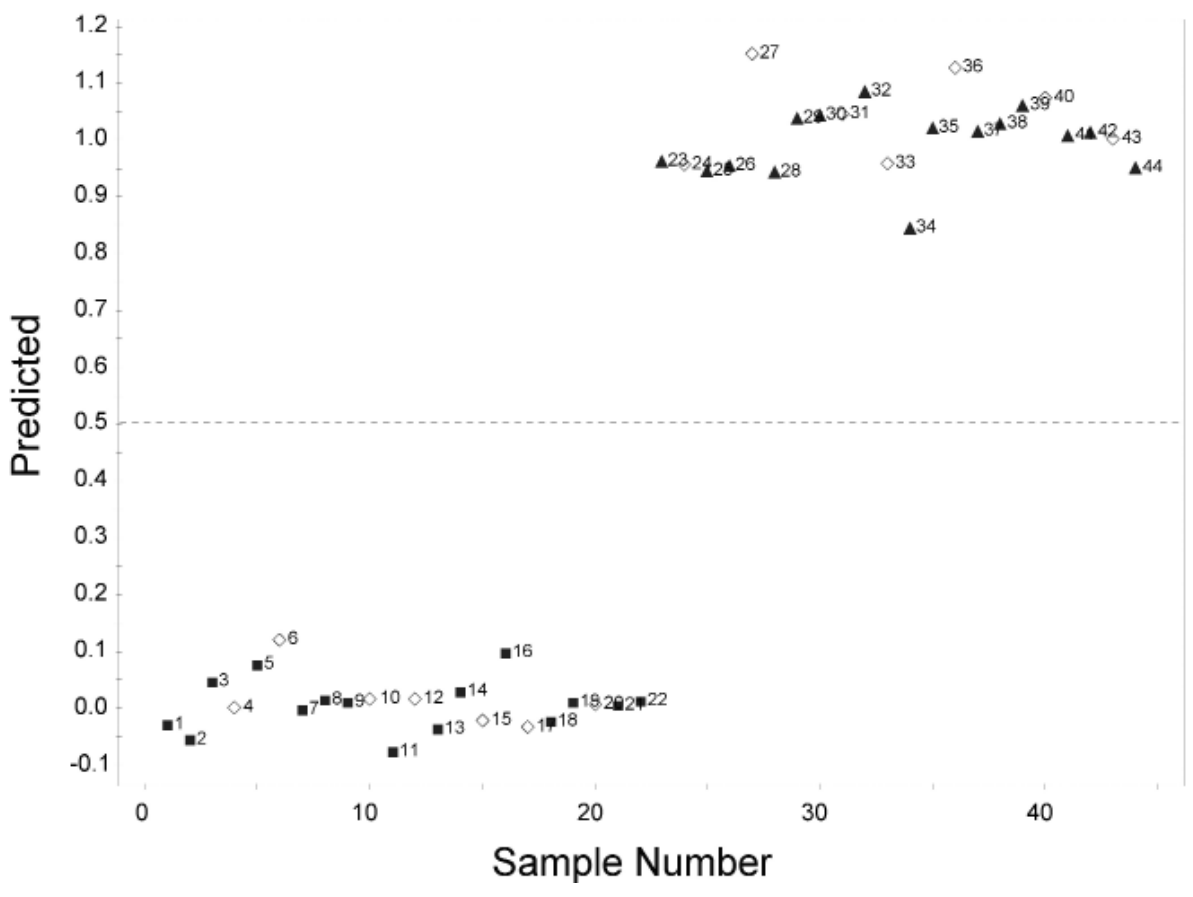

Fig. 6. Validation of the statistical model through prediction of unknown samples from non-treated and treated group. Filled box: non-treated group (training set), Filled triangle: treated group (training set), Open diamond: results for the blind test set. The dashed line represents the a priori cutoff $(0.5)$ for the class membership differentiation.
In the above literature, $100 \%, 93 \%$, and $70 \%$ accuracy was achieved for samples obtained at 28 days, 5 days, and 1 day time points, respectively. Still, the values may change a bit depending on which variables to use in the classification. In comparison, OPLS-DA approach does not depend on variable selection, as it uses all the variables at once for the classification. Moreover, it is straightforward to predict additional unknown samples using the established model. Therefore, OPLS-DA could be an objective and robust alternative in analyzing drug-induced toxicity (Clayton et al., 2006).

Recently, two studies employed metabolomics approach to establish early markers for kidney toxicity induced by cisplatin. In one study with NMR approaches on mice $(n=7$ per group, $20 \mathrm{mg} / \mathrm{kg}$ single dose) increased free fatty acid, amino acids and glucose in urine. In the other study $(n=6$ per treatment group, $0.5 \mathrm{mg} / \mathrm{kg}$ per day), GC-MS and LC-MS based approaches on SD rats found that amino acids are one of the most strongest markers for assessing early kidney toxicity. We performed NMR-based study on SD rats $(n=22,10 \mathrm{mg} /$ $\mathrm{kg}$ single dose) and found that dimethylurate, taurine, glycine, glucose and branched chain amino acids are elevated by the cisplatin treatment. Therefore, our results confirm the validity of the previously found markers and present additional markers with much larger number of animals. As the previous two results and ours employed different species (mouse vs. rat), cisplatin doses $(0.5 \mathrm{mg} / \mathrm{kg}$ qd, $10 \mathrm{mg} / \mathrm{kg}$, or $20 \mathrm{mg} / \mathrm{kg}$ ), and analytical techniques (Mass spectrometry vs. NMR), the consistent results of elevated levels of glucose and branched chain amino acids show that the metabolomics approach in assessing kidney toxicity is very robust, and should be widely applicable in other drug-induced toxicity studies.

\section{ACKNOWLEDGMENTS}

This work was supported by a grant of the Korea Health- care technology R\&D Project, Ministry for Health, Welfare \& Family Affairs, Republic of Korea (A084338).

\section{REFERENCES}

Arany, I. and Safirstein, R. L. (2003) Cisplatin nephrotoxicity. Semin. Nephrol. 23, 460-464.

Boudonck, K. J., Mitchell, M. W., Nemet, L., Keresztes, L., Nyska, A., Shinar, D. and Rosenstock, M. (2009) Discovery of metabolomics biomarkers for early detection of nephrotoxicity. Toxicol. Pathol. 37, 280-292.

Bylesjo, M., Rantalainen, M., Cloarec, O., Nicholson, J., Holmes, E. and Trygg, J. (2006) OPLS discriminant analysis: combining the strengths of PLS-DA and SIMCA classification. J. Chemom. 20, 341-351.

Clayton, T. A., Baker, D., Lindon, J. C., Everett, J. R. and Nicholson, J. K. (2009) Pharmacometabonomic identification of a significant host-microbiome metabolic interaction affecting human drug metabolism. Proc. Natl. Acad. Sci. USA. 106, 14728-14733.

Clayton, T. A., Lindon, J. C., Cloarec, O., Antti, H., Charuel, C., Hanton, G., Provost, J. P., Le Net, J. L., Baker, D., Walley, R. J., Everett, J. R. and Nicholson, J. K. (2006) Pharmaco-metabonomic phenotyping and personalized drug treatment. Nature 440, 1073-1077.

Cloarec, O., Dumas, M. E., Craig, A., Barton, R. H., Trygg, J., Hudson, J., Blancher, C., Gauguier, D., Lindon, J. C., Holmes, E. and Nicholson, J. (2005) Statistical total correlation spectroscopy: an exploratory approach for latent biomarker identification from metabolic $1 \mathrm{H}$ NMR data sets. Anal. Chem. 77, 1282-1289.

Crino, L., Calandri, C., Maestri, A. and Marrocolo, F. (2001) Gemcitabine and cisplatin combination in early-stage non-small-cell lung cancer. Oncology (Williston Park) 15, 40-42.

Davis, J. W. and Kramer, J. A. (2006) Genomic-based biomarkers of drug-induced nephrotoxicity. Expert Opin. Drug Metab. Toxicol. 2, 95-101.

Ebbels, T. M., Keun, H. C., Beckonert, O. P., Bollard, M. E., Lindon, J. C., Holmes, E. and Nicholson, J. K. (2007) Prediction and classification of drug toxicity using probabilistic modeling of temporal metabolic data: the consortium on metabonomic toxicology screening approach. J. Proteome. Res. 6, 4407-4422.

Gong, J. G., Costanzo, A., Yang, H. Q., Melino, G., Kaelin, W. G. Jr., 
Levrero, M. and Wang, J. Y. (1999) The tyrosine kinase c-Abl regulates p73 in apoptotic response to cisplatin-induced DNA damage. Nature 399, 806-809.

Hewitt, S. M., Dear, J. and Star, R. A. (2004) Discovery of protein biomarkers for renal diseases. J. Am. Soc. Nephrol. 15, 1677-1689.

Kang, J., Choi, M. Y., Kang, S., Kwon, H. N., Wen, H., Lee, C. H., Park M., Wiklund, S., Kim, H. J., Kwon, S. W. and Park, S. (2008a) Application of a $1 \mathrm{H}$ nuclear magnetic resonance (NMR) metabolomics approach combined with orthogonal projections to latent structurediscriminant analysis as an efficient tool for discriminating between Korean and Chinese herbal medicines. J. Agric. Food Chem. 56 11589-11595

Kang, J., Lee, S., Kang, S., Kwon, H. N., Park, J. H., Kwon, S. W. and Park, S. (2008b) NMR-based metabolomics approach for the differentiation of ginseng (Panax ginseng) roots from different origins. Arch. Pharm. Res. 31, 330-336.

Loehrer, P. J. and Einhorn, L. H. (1984) Drugs five years later. Cisplatin. Ann. Intern. Med. 100, 704-713.

Maher, A. D., Cloarec, O., Patki, P., Craggs, M., Holmes, E., Lindon, J. C. and Nicholson, J. K. (2009) Dynamic biochemical information recovery in spontaneous human seminal fluid reactions via $1 \mathrm{H}$ NMR kinetic statistical total correlation spectroscopy. Anal. Chem. 81, 288-295.

Maxuitenko, Y. Y., North, W. G. and Roebuck, B. D. (1997) Urinary taurine as a non-invasive marker of aflatoxin B1-induced hepatotoxicity: success and failure. Toxicology 118, 159-169.

Muggia, F. (2009) Platinum compounds 30 years after the introduction of cisplatin: implications for the treatment of ovarian cancer. Gynecol. Oncol. 112, 275-281.

Nicholson, J. K., Connelly, J., Lindon, J. C. and Holmes, E. (2002) Metabonomics: a platform for studying drug toxicity and gene function. Nat. Rev. Drug Discov. 1, 153-161.

Nicholson, J. K., Lindon, J. C. and Holmes, E. (1999) 'Metabonomics' understanding the metabolic responses of living systems to pathophysiological stimuli via multivariate statistical analysis of biologica NMR spectroscopic data. Xenobiotica. 29, 1181-1189.

Portilla, D., Li, S., Nagothu, K. K., Megyesi, J., Kaissling, B., Schnackenberg, L., Safirstein, R. L. and Beger, R. D. (2006) Metabolomic study of cisplatin-induced nephrotoxicity. Kidney International 69 2194-2204.

Pruefer, F. G., Lizarraga, F., Maldonado, V. and Melendez-Zajgla, J. (2008) Participation of Omi Htra2 serine-protease activity in the apoptosis induced by cisplatin on SW480 colon cancer cells. J. Chemother. 20, 348-354

Rozen, R., Tenenhouse, H. S. and Scriver, C. R. (1979). Taurine trans- port in renal brush-border-membrane vesicles. Biochem. J. 180, 245-248.

Sands, C. J., Coen, M., Maher, A. D., Ebbels, T. M., Holmes, E., Lindon, J. C. and Nicholson, J. K. (2009) Statistical total correlation spectroscopy editing of $1 \mathrm{H}$ NMR spectra of biofluids: application to drug metabolite profile identification and enhanced information recovery. Anal. Chem. 81, 6458-6466.

Smith, I. E. and Talbot, D. C. (1992) Cisplatin and its analogues in the treatment of advanced breast cancer: a review. Br. J. Cancer 65, 787-793

Sreekumar, A., Poisson, L. M., Rajendiran, T. M., Khan, A. P., Cao, Q., Yu, J., Laxman, B., Mehra, R., Lonigro, R. J., Li, Y., Nyati, M. K., Ahsan, A., Kalyana-Sundaram, S., Han, B., Cao, X., Byun, J., Omenn, G. S., Ghosh, D., Pennathur, S., Alexander, D. C., Berger, A., Shuster, J. R., Wei, J. T., Varambally, S., Beecher, C. and Chinnaiyan, A. M. (2009) Metabolomic profiles delineate potential role for sarcosine in prostate cancer progression. Nature. 457, 910-914.

Trygg, J. and Wold, S. (2002) Orthogonal projections to latent structures (O-PLS). J. Chemom. 16, 119-128.

von der Maase, H., Hansen, S. W., Roberts, J. T., Dogliotti, L., Oliver, T., Moore, M. J., Bodrogi, I., Albers, P., Knuth, A., Lippert, C. M., Kerbrat, P., Sanchez Rovira, P., Wersall, P., Cleall, S. P., Roychowdhury, D. F., Tomlin, I., Visseren-Grul, C. M. and Conte, P. F. (2000) Gemcitabine and cisplatin versus methotrexate, vinblastine, doxorubicin, and cisplatin in advanced or metastatic bladder cancer: results of a large, randomized, multinational, multicenter, phase III study. J. Clin. Oncol. 18, 3068-3077.

Wen, H., Kang, S., Song, Y., Sung, S. H. and Park, S. (2010a) Differentiation of cultivation sources of Ganoderma lucidum by NMR-based metabolomics approach. Phytochem. Anal. 21, 73-79.

Wen, H., Yoo, S. S., Kang, J., Kim, H. G., Park, J. S., Jeong, S., Lee, J. I., Kwon, H. N., Kang, S., Lee, D. H. and Park, S. (2010b) A new NMR-based metabolomics approach for the diagnosis of biliary tract cancer. J. Hepatol. 52, 228-233.

Wiklund, S., Johansson, E., Sjostrom, L., Mellerowicz, E. J., Edlund U., Shockcor, J. P., Gottfries, J., Moritz, T. and Trygg, J. (2008) Visualization of GC/TOF-MS-based metabolomics data for identification of biochemically interesting compounds using OPLS class Models. Anal. Chem. 80, 115-122.

Wishart, D. S. (2005) Metabolomics: the principles and potential applications to transplantation. Am. J. Transplant. 5, 2814-2820.

Yao, X., Panichpisal, K., Kurtzman, N. and Nugent, K. (2007) Cisplatin nephrotoxicity: a review. Am. J. Med. Sci. 334, 115-124. 